\title{
Correcting and sharing our complications. Misplacement of pigtail catheter, during a Robot Assisted Pyeloplasty. Clinical findings, diagnosis, possible causes and endoscopic treatment
}

\author{
Konstantinos Stravodimos ${ }^{1}$, Ioannis Katafigiotis ${ }^{2}$, Evangelos Fragkiadis ${ }^{3}$, Stavros Tyritzis ${ }^{3}$, \\ Constantinos A. Constantinides ${ }^{4}$ \\ $1^{\text {st }}$ University Urology Clinic, Laiko Hospital, Greece.
}

\begin{abstract}
Summary Objective: Robotic assisted pyeloplasty $(R A P)$ is rapidly adopted by surgeons around the world. We present a unique complication of the technique, consisting of pigtail misplacement, which was endoscopically resolved. We discuss the clinical findings, differential diagnosis and principles of endoscopic treatment. Materials and Methods: A 41 years old female patients underwent transperitoneal right side RAP with the Hynes-Anderson technique for ureteropelvic junction obstruction. Pigtail was placed intraoperatively in an antegrade fashion. Post operative course appeared normal but Kidney-Ureterer-Bladder(KUB) $X$-ray, revealed a misplaced pigtail. Patient underwent a semirigid ureterorenoscopy demonstrating that the pigtail was exiting the collecting system in the rear line of suturing between continuous sutures. Pigtail was retrieved with a stone retrieval forceps with short upward motions in the renal pelvis under fluoroscopy and then removed from patient, in order to avoid stressing the anastomosis. No leakage was noted in fluoroscopy, a pigtail was correctly placed and patient recovery was uneventful.

Results: Retrograde pyelography was the key to accurate diagnosis and endoscopic treatment, because the exact point of exit and anastomosis integrity were established. Retrieval of the pigtail was the most challenging part. Lack of proper visualization and mobilization of the rear part of the anastomosis during surgery, combined with lack of tactile feedback,

because of robotic instrumentation, were of critical importance in the manifestation of such a mishap. Endoscopy facilitated case resolve, but proper handling is required to protect the anastomosis.

Conclusions: The introduction of novel techniques can carry the burden of novel complications. A surgeon must always keep in mind the complications inherent to the technique and at the same time the limitations of the equipment used, especially the lack of tactile feedback in robotic instrumentation.
\end{abstract}

KEY WORDS: Robotic assisted pyeloplasty; Misplaced pig tail; Pyeloplasty complication.

Submitted 1 December 2014; Accepted 28 January 2015

\section{INTRODUCTION}

Robot Assisted Pyeloplasty (RAP) constitutes a less invasive but similar safe and effective method compared to open pyeloplasty for the surgical management of the ureteropelvic junction obstruction (UPJO). The good efficacy of the method, the excellent surgeon control of dissection and suturing, combined with the fast recovery, minimal pain and good aesthetic results may explain the rapid diffusion of the robotic approach (1-3). The introduction of novel techniques can carry the burden of novel complications. A surgeon must always keep in mind the complications inherent to the technique and at the same time the limitations of the equipment used, especially the lack of tactile feedback in robotic instrumentation.

\section{Case presentation}

A female patient aged 41 was subjected to robot assisted transperitoneal right dismembered Hynes-Anderson pyeloplasty, after excision of the stenotic part of the UPJ. A pigtail was placed in an antegrade fashion over a PTFE guidewire after the completion of the rear part of the anastomosis with a continuous PDS suture 4.0. After the pigtail placement the anastomosis was completed, a drain was placed near the UPJ area through a robotic port. Patient recovered normally from general anaesthesia and was transferred to the Urology ward.

Post operative course was uneventful. Patient was mobilized in the same evening and received a light supper and fluids. Standard use of paracetamol as analgesic was adequate for managing patient post-operative pain.

Drainage fluid collection was minimal (less than $50 \mathrm{cc}$ ) in the first 24 hours. Clinical examination was normal with normal abdomen sounds and no signs of peritoneal distress in abdomen palpation. In the first postoperative day a KUB X-ray was performed as standard care to confirm the correct placement of the stent (Figure 1).

The Kidney-Ureter-Bladder (KUB) x-ray showed a rather complicating picture in which the upper part-tail of the stent seemed to be in correct position inside the renal pelvis but the lower part was misplaced. An emergency ultrasound following the KUB X-ray, confirmed that the upper part of the pigtail was placed inside the renal pelvis while was negative for urinoma. Pigtail end was not found inside the bladder. 


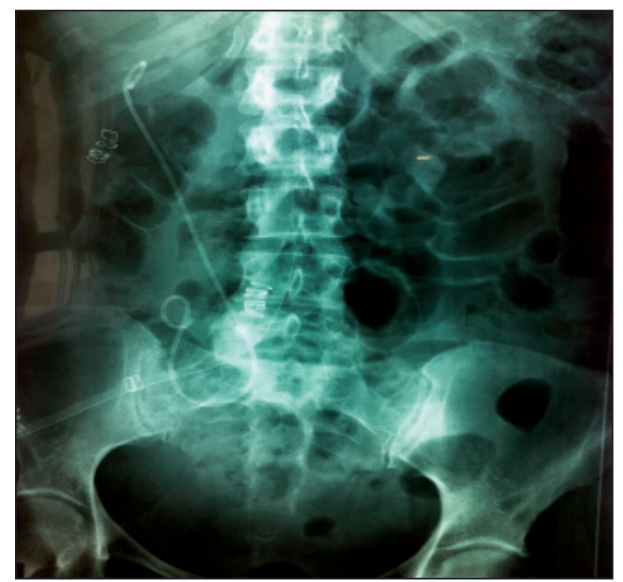

Figure 1.

KUB X-ray

Depicting the

misplacement

of the Pig-Tail.

Figure 2.

Semirigid ureteroscopy and retrieval of the pig tail inside the renal pelvis.

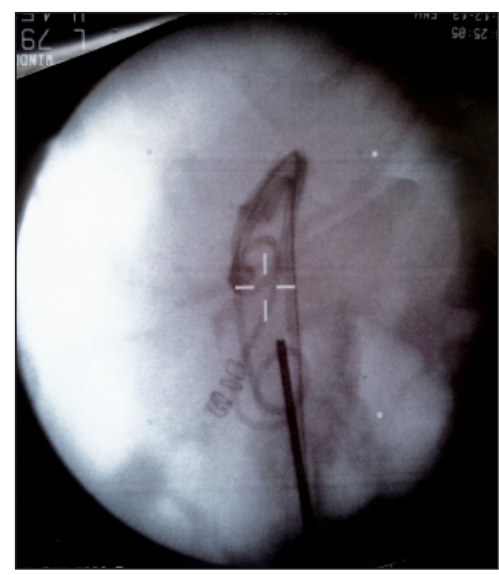

Figure 3.

Fluoroscopy depicting minor leakage of the ureteropelvic anastomosis

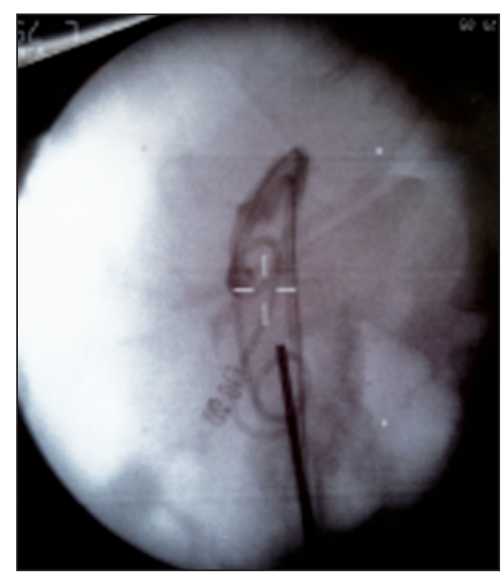

reached the urinary bladder, the right orifice was easily recognized and catheterized. Retrograde pyelography was performed with fluoroscopy, no sign of leakage was noticed, while only the initial upper part of the pigtail was in the renal pelvis. A hydrophilic Zip-wire was easily placed as a safety wire and a PTFE wire as guidewire. Semirigid ureteroscopy over the guidewire was performed all the way up to the renal pelvis (Figure 2.). At the rear part of the anastomosis in between the suturing line, the pigtail was recognized exiting the urinary tract. After correct diagnosis the next step was to remove the misplaced pigtail. In order to remove the pigtail we chose to handle it not from the tip but exactly from the site that was exiting the anastomosis using a stone retrieval forceps. Slow short curved moves were used to tract the pigtail backwards and upwards inside the renal pelvis, so as to put minimal tension on the anastomosis under simultaneous optical and fluoroscopy guidance. After complete retrieval of the stent inside the urinary tract the tip of the pigtail was pulled again with the forceps and removed with the ureteroscope. A second look retrograde pyelography revealed minor leakage and a new pigtail was placed under fluoroscopy (Figure 3). Patient had no drain output during the next 24 hours, no pain, normal bowel movement and she was discharged the following day. A new Intravenous pyelography was performed as a follow-up two months later depicting an intact pyeloureteric anastomosis

Discussion and supplementary references are posted on www.aiua.it

\section{Differential diagnosis}

The differential diagnosis included three case scenarios. Initial concern was that the guidewire during the placement either never reached the bladder and as a result the pigtail was not properly placed in the bladder but the whole lower part and the tip of course curled inside the ureter. Another possibility was that during the insertion of the pigtail, even though we used the flexible tip inside the urinary tract, a perforation had occurred and as a result the pigtail was exiting the urinary tract through the perforation of the ureter inside the abdomen. The last but the worst case scenario was that the double J was misplaced at the initial placement in the anastomosis, thus meaning that during the insertion the guidewire not only didn't entered the ureter but was moving freely inside the abdomen cavity with the risk of traumatizing a vital organ-tissue. Our primary concerns were to place a new pigtail in a proper position to establish normal urine drainage and to remove the misplaced pigtail without compromising the integrity of the anastomosis and the ureter or risking any abdomen organ injury. We opted for an endoscopic approach for optimum diagnosis and the possibility of final resolution.

\section{Endoscopy treatment}

Cystoscopy, as expected, revealed that the pigtail had not

\section{References}

1. Autorino R, Eden C, El-Ghoneimi A, et al. Robot-assisted and laparoscopic repair of ureteropelvic junction obstruction: a systematic review and meta-analysis. Eur Urol. 2014; 65:430-52.

2. Mendez-Torres F, Woods M, Thomas R. Technical modifications for robot-assisted laparoscopic pyeloplasty. J Endourol. 2005; 19:393-6.

3. Sethi AS, Regan SM, Sundaram CP. Robot-assisted laparoscopic pyeloplasty with and without a ureteral stent. J Endourol. 2011; 25:239-43.

\section{Correspondence}

Konstantinos Stravodimos, MD Associate Professor kgstravod@yahoo.com

Ioannis Katafigiotis, MD (Corresponding Author) Resident katafigiotis@yahoo.com

Evangelos Fragkiadis, MD Associate Scientist-Urologist. e.fragkiadis@gmail.com

Stavros Tyritzis, MD Associate Scientist-Urologist statyr@freimail.gr

Constantinos A. Constantinides, MD Professor of Urology ckonstan@med.uoa.gr

$1^{\text {st }}$ University Urology clinic, Athens University Medical School Laiko Hospital, 17 Agiou Thoma str., 11527, Athens, Greece 